International Journal of Pure and Applied Mathematics

Volume 115 No. 1 2017, 13-25

ISSN: 1311-8080 (printed version); ISSN: 1314-3395 (on-line version)

url: http://www.ijpam.eu

doi: 10.12732 /ijpam.v115i1.2

\title{
A NOTE ON UNSTEADY HYDROMAGNETIC FLOW DUE TO TANGENTIAL STRESS AT THE HORIZONTAL FREE SURFACE
}

\author{
K. Jagadeshkumar ${ }^{1}$, Vempaty Somaraju ${ }^{2}$, S. Srinivas ${ }^{1}$ \\ ${ }^{1}$ Department of Mathematics \\ School of Advanced Sciences \\ VIT University, Vellore, Tamil Nadu, INDIA \\ ${ }^{2}$ Department of Mathematics,LIAS \\ GVP College of Engineering (Autonomous) \\ Vishakhapatnam, Andhra Pradesh, INDIA
}

\begin{abstract}
The problem of unsteady unidirectional hydromagnetic flow due to stress applied at the free surface is studied here. The applied magnetic field is normal to the horizontal boundary. The dynamics of fluid and electric currents are investigated as functions of magnetic Prandtl number $P_{m}\left(=\sigma \mu_{0} \nu\right)$, which measures the ratio of viscous diffusion to magnetic diffusion. In contrast to hydrodynamic Rayleigh problem, the transient dynamics consists of two diffusively growing layers as in the conventional MHD Rayleigh problem (see Dix [5]). The viscous Hartmann layer becomes steady soon, while the magnetic diffusion layer diffuses to infinity with Alfven speed. The electric currents generated in the Hartmann layer find a return path in the magnetic diffusion layer. It may also be seen that the free surface Hartmann layer is weak compared to rigid surface Hartmann layer.
\end{abstract}

AMS Subject Classification: $76 \mathrm{~W}$

Key Words: magnetohydrodynamics, Hartmann layer, magnetic Prandtl number

\section{Introduction}

The classical Rayleigh's problem studies the motion of an infinitely extended,

Received: December 15, 2016

Revised: $\quad$ May 30, 2017

Published: $\quad$ June 24, 2017

(C) 2017 Academic Publications, Ltd. url: www.acadpubl.eu

$\S_{\text {Correspondence author }}$ 
incompressible, viscous fluid in response to an infinite flat plate suddenly set in motion along its own plane. The usefulness of such a problem is that it exhibits qualitative boundary layer characteristics. In fact, immediately following the impulse, a simple Rayleigh layer, unaffected by other affects such as rotation and magnetic field, develops with a dimensional thickness of $O(\nu t)^{\frac{1}{2}}$. Batchelor [1] considered the Rayleigh problem for a free surface subject to a constant stress and derived the solution for the unidirectional velocity field $u(y, t)$, and $\frac{\partial u}{\partial y}$. As Batchelor mentioned, these solutions, under suitable conditions, correspond to the velocity distribution in water set into motion by air blowing steadily over its free surface. Astrogeophysical flow systems are seldom undisturbed and so extensions to classical Rayleigh problem including other affects invite our attention. It is well known that the inclusion of Coriolis force in Rayleigh problem makes the flow steady and confines the velocity distribution to a boundary layer, known as Ekman layer. Batchelor [1] (also see Pedlosky [7]) presented the analysis of steady Ekman layer at a free surface. Similarly, there exist magnetohydrodynamic extensions of Rayleigh problem with a mechanical forcing on rigid boundary (for ex. Hughes and Young [6]).Following Batchelor's analysis of Rayleigh problem for a free surface, we wish to analyze unsteady hydromagnetic flow due to a tangential stress applied at the free surface of a conducting fluid. The ultimate aim is to understand free surface rotating bounded (and unbounded) hydromagnetic flows including the effect of variation of Coriolis parameter.

Rossow [8], Dix [5], and several others have attacked the hydromagnetic Rayleigh problem from different angles. We wish to extend the Hydromagnetic Rayleigh problem analysis as given by Dix [5], and Hughes \& Young [6] for a rigid surface to a free surface. The solution of the linear problem will be studied using Laplace transform technique. All possible limiting cases will be discussed.

The statement of the problem and mathematical formulation are given in II. Exact Laplace transform solution followed by limiting cases of interest are discussed in III. Section IV deals with a special case of interest for which the magnetic prandtl number is unity. The physically important case of non-vanishing but small magnetic prandtl number is presented in V. Finally the results are summarized in VI.

\section{Statement of the Problem}

We consider an infinite expanse of an incompressible conducting fluid initially at rest. The fluid is bounded by a horizontal free surface at the top at $y=0$. 
A uniform magnetic field of strength $\vec{B}_{0}$ is applied parallel to the y-axis. At timet $=0$, a uniform and constant stress $\frac{A^{2}}{\nu} \tau_{0}$ is applied at the free surface in $\mathrm{x}$-direction (see Fig.1) where $\mathrm{A}$ is the Alfven speed and $\nu$ is the kinematic viscosity.
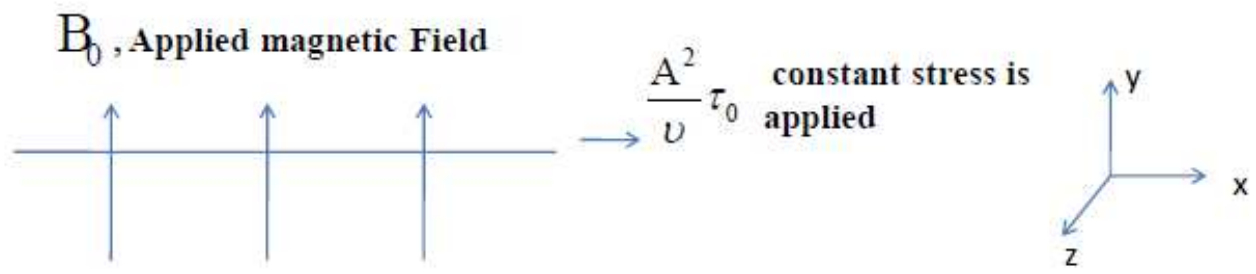

Figure 1: Hydromagnetic Rayleigh problem at a free surface

The governing equations are the same as those given by Hughes \& Young [6]. This is a problem of unidirectional flow with $\vec{V}=u(y, t) \hat{i}$ and induced magnetic field $h(y, t)$ in the y-direction. The governing equations may be written as (see Hughes \& Young [6])

$$
\begin{gathered}
\rho \frac{\partial u}{\partial t}=\mu_{f} \frac{\partial^{2} u}{\partial y^{2}}+B_{0} \frac{\partial h}{\partial y} \\
\frac{\partial^{2} h}{\partial y^{2}}=\sigma \mu_{0} \frac{\partial h}{\partial t}-\sigma B_{0} \frac{\partial u}{\partial y}
\end{gathered}
$$

where $\rho$ is fluid density, $\mu_{f}$ is fluid viscosity, $\sigma$ and $\mu_{0}$ are conductivity and magnetic permeability of the fluid. Fluid motions are driven by the constant stress applied along the direction $x$-axis as mentioned earlier. The initial and boundary conditions will be directly presented after non-dimensionalization. There is no characteristic length scale in the problem. The length scale may be chosen based on Alfven speed to avoid one nondimensional number. Following Hughes \& Young [6], we substitute the following dimensionless starred variables, into (1)-(2)

$$
\begin{aligned}
& -y^{*}=\frac{A y}{v}, z^{*}=\frac{A z}{v}, t^{*}=\frac{A^{2} t}{v} \\
& h^{*}=\frac{h}{H_{0}}, u^{*}=\frac{u}{A}, G^{*}=\frac{v}{\rho A^{3}} \frac{\partial p}{\partial x}
\end{aligned}
$$

where $A=\frac{B_{0}}{\sqrt{\rho \mu_{0}}}$ is the Alfven speed. It may be noted that as $y$ goes from 0 to 
$-\infty$, the variable $y^{*}$ goes from 0 to $\infty$. We now obtain,

$$
\begin{gathered}
\frac{\partial^{2} u^{*}}{\partial y^{* 2}}-\frac{\partial h^{*}}{\partial y^{*}}=\frac{\partial u^{*}}{\partial t^{*}} \\
\frac{\partial^{2} h^{*}}{\partial y^{* 2}}-p_{m} \frac{\partial u^{*}}{\partial y^{*}}=p_{m} \frac{\partial h^{*}}{\partial t^{*}}
\end{gathered}
$$

where $p_{m}=\sigma \mu_{0} \nu$ is magnetic Prandtl number. Boundary conditions in nondimensional form may be written as,

$$
\left.\begin{array}{ccc}
\frac{\partial u^{*}}{\partial y^{*}}\left(t^{*}, 0\right)=-\tau_{0}, & u^{*}\left(t^{*}, \infty\right)=0, & u^{*}(0, y)=0 \\
h^{*}\left(t^{*}, \infty\right)=0, & h^{*}\left(t^{*}, 0\right)=0, & h^{*}(0, y)=0
\end{array}\right\}
$$

\section{Transient Solution and Limiting Cases}

Taking Laplace transform of (4)-(6) with the respect to dimensionless time, we get

$$
\begin{gathered}
\frac{d^{2} \bar{u}^{*}}{d y^{*^{2}}}-s \bar{u}^{*}=\frac{d \bar{h}^{*}}{d y^{*}} \\
\frac{d^{2} \bar{h}^{*}}{d y^{*^{2}}}-p_{m} s \bar{h}^{*}=p_{m} \frac{d \bar{u}^{*}}{d y^{*}}
\end{gathered}
$$

The boundary conditions in transform plane become:

$$
\left.\begin{array}{ccc}
\frac{\partial \bar{u}^{*}}{\partial y^{*}}(s, 0)=-\frac{\tau_{0}}{s}, & \bar{u}^{*}(s, \infty)=0, & \bar{u}^{*}(0, y)=0 \\
\bar{h}^{*}(s, \infty)=0, & \bar{h}(s, 0)=0, & \bar{h}^{*}(0, y)=0
\end{array}\right\}
$$

The equations (7) and (8) can be combined to give,

$$
\left[\frac{d^{4}}{d y^{4}}-\left[s\left(1+p_{m}\right)+p_{m}\right] \frac{d^{2}}{d y^{2}}+p_{m} s^{2}\right]\left(\begin{array}{l}
\bar{u}^{*} \\
\bar{h}^{*}
\end{array}\right)=0
$$

Using boundary conditions at $y^{*}=\infty$, the solutions of $u^{*}$, and $h^{*}$ can be written as,

$$
\begin{gathered}
\bar{u}^{*}\left(s, y^{*}\right)=c_{1} e^{-\lambda_{1} y^{*}}+c_{2} e^{-\lambda_{2} y^{*}} \\
\bar{h}^{*}\left(s, y^{*}\right)=\frac{\left(\lambda_{1}^{2}-s\right)}{\lambda_{1}} c_{1} e^{-\lambda_{1} y^{*}}+\frac{\left(\lambda_{2}^{2}-s\right)}{\lambda_{2}} c_{2} e^{-\lambda_{2} y^{*}}
\end{gathered}
$$


where $\lambda_{1,2}=\frac{1}{2}\left[\sqrt{p_{m}+s\left(1+\sqrt{p_{m}}\right)^{2}} \pm \sqrt{p_{m}+s\left(1-\sqrt{p_{m}}\right)^{2}}\right]$. The constants $c_{1}$ and $c_{2}$ can be determined using the boundary conditions. They are,

$$
c_{1}=\frac{\tau_{0}\left(s-\lambda_{2}^{2}\right) \lambda_{1}}{D}, \quad c_{2}=-\frac{\tau_{0}\left(s-\lambda_{1}^{2}\right) \lambda_{2}}{D}
$$

where

$$
D=s^{2}\left(\lambda_{2}^{2}-\lambda_{1}^{2}\right)=-s^{2}\left(\sqrt{p_{m}+s\left(1-\sqrt{p_{m}}\right)^{2}}\right)\left(\sqrt{p_{m}+s\left(1+\sqrt{p_{m}}\right)^{2}}\right)
$$

Therefore, the solution of $\bar{u}, \bar{h}$ are,

$$
\begin{gathered}
\bar{u}^{*}=-\frac{\tau_{0}}{D}\left[\left(s-\lambda_{2}^{2}\right) \lambda_{1} e^{-\lambda_{1} y^{*}}-\left(s-\lambda_{1}^{2}\right) \lambda_{2} e^{-\lambda_{2} y^{*}}\right] \\
\bar{h}^{*}=-\frac{\tau_{0}\left(s-\lambda_{1}^{2}\right)\left(s-\lambda_{2}^{2}\right)}{D}\left[e^{-\lambda_{1} y^{*}}-e^{-\lambda_{2} y^{*}}\right]
\end{gathered}
$$

These solutions differ considerably from those of rigid surface Rayleigh problem. Yet, the physical features do not change much qualitatively. It may be noted from (13) that the flow has a double layer exponential structure in $y^{*}$. Following Benton \& Loper [2] these layers may be referred to as the $k$ and $m$ layers corresponding to the roots $\lambda_{1}$ and $\lambda_{2}$ respectively. From our previous knowledge $[2,6]$ the $k$ layer corresponds to Hartmann layer and the $m$ layer corresponds to magnetic diffusion layer or Alfven layer. Since for large time, $\lambda_{1}=\sqrt{P_{m}}$ , the thickness of the $k$ layer is $M^{-1}$ where $M=L B_{0} \sqrt{\frac{\sigma}{\mu}}$ is the Hartmann number. Exact inversion of (13) - (14) is not possible. We shall first present some interesting limiting cases of interest.

\subsection{Laplace Inversion for Some Limiting Cases}

\subsubsection{Steady State Solution (The $k$-Layer (Hartmann Layer) Dynamics)}

Since we expect a steady state, we may use the final value theorem,

$$
f(y, \infty)=s F(y, s) \text { as } s \rightarrow 0
$$

As $s \rightarrow 0$, we have, $\lambda_{1}=\sqrt{P_{m}}, \lambda_{2}=0, D=-s^{2} P_{m}$. The steady state solutions are,

$$
u^{*}=\frac{\tau_{0}}{\sqrt{P_{m}}} e^{-\sqrt{P_{m}} y^{*}}, \quad h^{*}=\left(e^{-\sqrt{P_{m}} y^{*}}-1\right)\left(-\tau_{0}\right)
$$


If we write in dimensional form, we shall realize that the solutions represent actually Hartmann layer and it is weak compared to rigid surface Hartmann layer. The fact that $\lambda_{2}$ clearly shows that the m-layer diffuses out to spatial infinity leaving out steady state k-layer. The velocity and magnetic field profiles in Hartmann layer ( $k$ layer) are shown below when $P m=1$.

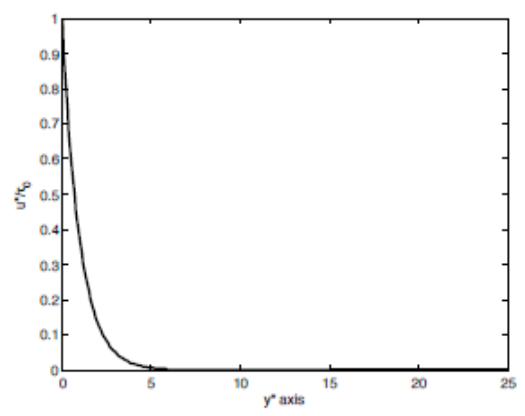

Figure 2: k layer velocity profile when $P_{m}=1$.

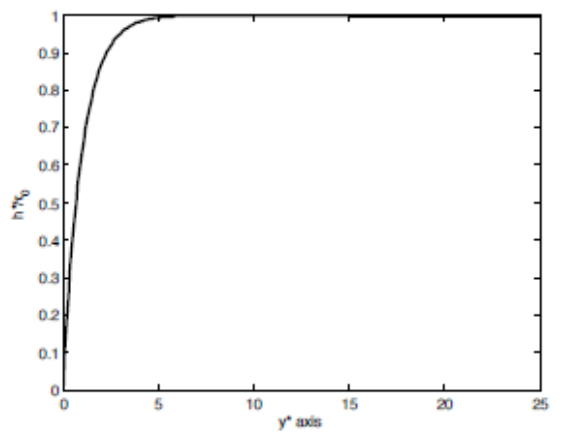

Figure 3: k layer magnetic field profile when $P_{m}=1$.

\subsubsection{The Early Time Behaviour}

The early time behavior of any of the functions in physical space is determined by the corresponding behavior of the transformed functions for large $\mathrm{s}$. The initial value theorem states

$$
f(y, 0)=s F(y, s) \text { as } s \rightarrow \infty
$$

The velocity $\bar{u}^{*}$ in such a case reduces to

$$
\bar{u}^{*}=\frac{\tau_{0}}{s^{3 / 2}}\left[e^{-\sqrt{s} y^{*}}\right]
$$

The inverse Laplace transform of (16) is (ref. Campbell \& Foster [3], table no. $824.5)$,

$$
u^{*}\left(y^{*}, t^{*}\right)=-\tau_{0} y^{*}+\tau_{0}\left[\sqrt{\frac{4 \nu t^{*}}{\pi}} \exp \left(-\frac{y^{* 2}}{4 \nu t^{*}}\right)+y^{*} \operatorname{erf}\left(\frac{y^{*}}{\sqrt{4 \nu t^{*}}}\right)\right]
$$

Equation (17) agrees with the classical Hydrodynamic Rayleigh problem for a free surface discussed by Batchelor [1]. Physically, we expect the simple Rayleigh layer solution immediately following the impulse. 


\subsubsection{Hydrodynamic Rayleigh Problem for a Free Surface $\left(P_{m}=0\right)$}

This limit $P_{m}=0$ is singular since it multiplies the time derivative terms in (5). In this limit, the equations reduce to

$$
\frac{\partial^{2} u^{*}}{\partial y^{* 2}}+\frac{\partial h^{*}}{\partial y^{*}}=\frac{\partial u^{*}}{\partial t^{*}} \quad \text { and } \quad \frac{\partial^{2} h^{*}}{\partial y^{*^{2}}}=0
$$

Therefore we get

$$
\frac{\partial^{2}\left(D_{1} u^{*}\right)}{\partial y^{*^{2}}}=\frac{\partial\left(D_{1} u^{*}\right)}{\partial t *} \text { where } D_{1} u^{*}=\frac{\partial u^{*}}{\partial y^{*}}
$$

Hence, $D_{1} u^{*}=-\tau_{0}+\tau_{0} \operatorname{erf}\left(y^{*} / 4 t^{*}\right)^{\frac{1}{2}}$.In fact, it is easily confirmed from (13) that,

$$
\bar{u}^{*}=\frac{\tau_{0}}{s^{3 / 2}}\left[e^{-\sqrt{s} y^{*}}\right]
$$

The inverse Laplace transform of (18) is (ref. Campbell \& Foster [3], table no. $824.5)$

$$
u^{*}\left(y^{*}, t^{*}\right)=-\tau_{0} y^{*}+\tau_{0}\left[\sqrt{\frac{4 \nu t^{*}}{\pi}} \exp \left(-\frac{y^{* 2}}{4 \nu t^{*}}\right)+y^{*} \operatorname{erf}\left(\frac{y^{*}}{\sqrt{4 \nu t^{*}}}\right)\right]
$$

Equation (19) and the solution for $D_{1} u^{*}$ agree with the classical Hydrodynamic Rayleigh problem for a free surface discussed by Bathcelor [1]. In fact the limit $\mathrm{Pm}=0$ in the present case shows that there cannot be a Hartmann layer at the free surface since $\frac{d^{2} h^{*}}{d y^{* 2}}$. The $m$ layer (Magnetic diffusion layer) is suppressed in this case since it diffuses instantaneously to infinity and becomes spatially uniform. As a result we have only the hydrodynamic Rayleigh layer solution given by (19). Thus for $P_{m} \rightarrow 0$, the hydromagnetic effects become unimportant in our problem.

\section{Solution of the Problem when $P_{m}=1$}

In this case we assume that the Prandtl number is unity. The values of $\lambda_{1}, \lambda_{2}, D, c_{1}$, and $c_{2}$ in this limiting case are,

$$
\left.\begin{array}{l}
\lambda_{1}=\frac{1}{2}[\sqrt{1+4 s}+1] ; \lambda_{2}=\frac{1}{2}[\sqrt{1+4 s}-1] \\
D=-s^{2}(\sqrt{1+4 s}), c_{1}=-\frac{\tau_{0}}{s(\sqrt{1+4 s})} ; c_{2}=\frac{\tau_{0}}{s(\sqrt{1+4 s})}
\end{array}\right\}
$$


Using (20), the expressions for velocity, induced magnetic field and current density are:

$$
\begin{aligned}
& \bar{u}^{*}=\frac{\tau_{0}}{2 s\left(\sqrt{s+\frac{1}{4}}\right)}\left[e^{-\frac{y^{*}}{2}} e^{-\sqrt{s+\frac{1}{4}} y^{*}}+e^{\frac{y^{*}}{2}} e^{-\sqrt{s+\frac{1}{4}} y^{*}}\right] \\
& \bar{h}^{*}=\frac{\tau_{0}}{2 s\left(\sqrt{s+\frac{1}{4}}\right)}\left[e^{-\frac{y^{*}}{2}} e^{-\sqrt{s+\frac{1}{4}} y^{*}}-e^{\frac{y^{*}}{2}} e^{-\sqrt{s+\frac{1}{4}} y^{*}}\right]
\end{aligned}
$$

These solutions are slightly different from the rigid surface hydromagnetic Rayleigh problem. We now have an additional term $\left(s+\frac{1}{4}\right)^{\frac{1}{2}}$ in the denominator. However, for large times $s \rightarrow 0$, this term reduces to $\frac{1}{2}$. The inverse transform of (21)-(22) are, (ref. Campbell \& Foster [3], table no. 825)

$$
\begin{aligned}
& u^{*}\left(y^{*}, t^{*}\right)=\frac{\tau_{0}}{2}\left[-\operatorname{erfc}\left[\frac{y^{*}+t^{*}}{2 \sqrt{t^{*}}}\right]\left(1+e^{y^{*}}\right)+\operatorname{erfc}\left[\frac{y^{*}-t^{*}}{2 \sqrt{t^{*}}}\right]\left(1+e^{-y^{*}}\right)\right] \\
& h^{*}\left(y^{*}, t^{*}\right)=\frac{\tau_{0}}{2}\left[\operatorname{erfc}\left[\frac{y^{*}+t^{*}}{2 \sqrt{t^{*}}}\right]\left(1-e^{y^{*}}\right)-\operatorname{erfc}\left[\frac{y^{*}-t^{*}}{2 \sqrt{t^{*}}}\right]\left(e^{-y^{*}}-1\right)\right]
\end{aligned}
$$

It can be seen from (23) and (24) that the flow consist of two diffusing Alfven waves having decaying amplitude (because of $2 \sqrt{t}$ in the denominator). Both these backward and forward waves decay due to viscous dissipation. The backward wave term has significant magnitude only near to the boundary and it is necessary to satisfy the boundary condition. At the free surface viscous forces are not strong. So, the Hartmann boundary layer thickness is relatively large compared to the one at the rigid surface. The solutions for $u^{*}$ and $h^{*}$ given by (23) and (24) are shown below in Figure (4).

\subsection{Large Time Behavior of Solutions when $P_{m}=1$}

Now, we wish to examine the solutions for large values of time. Allowing $s \ll 1$ in (21)-(22) and using the relation $j_{z}=-\frac{\partial h_{x}}{\partial y}$, we get

$$
\left.\begin{array}{l}
\bar{u}^{*}=\frac{\tau_{0}}{s}\left[e^{-y^{*}}+1\right] e^{-y^{*} s} \\
\bar{h}^{*}=\frac{\tau_{0}}{s}\left[e^{-y^{*}}-1\right] e^{-y^{*} s} \\
\bar{j}^{*}=\frac{\partial h_{x}^{*}}{\partial y^{*}}=\frac{\tau_{0}}{s}\left[-e^{-y^{*}}+s\right] e^{-y^{*} s}
\end{array}\right\}
$$



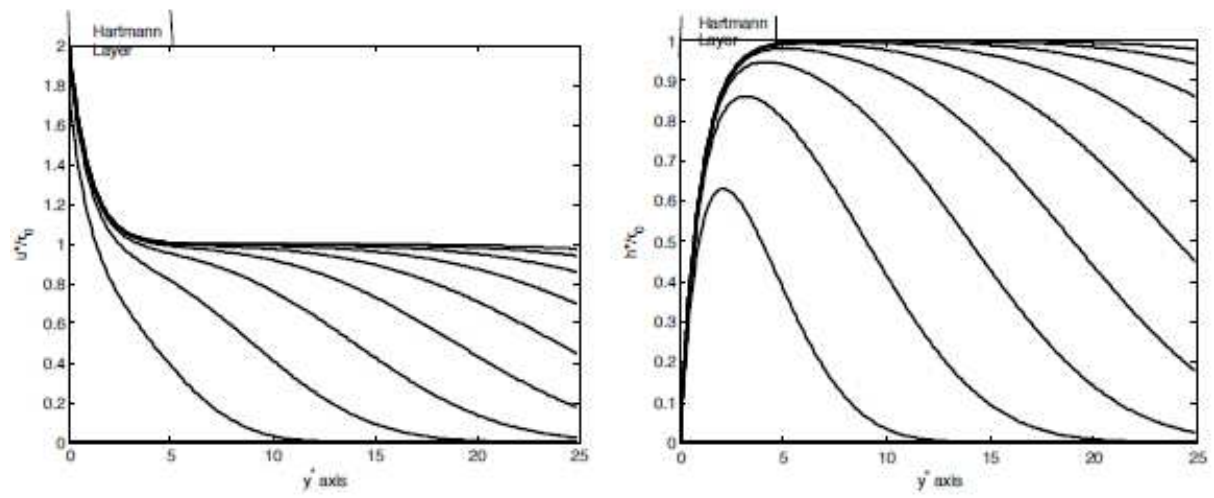

Figure 4: (a) velocity distribution for various values of time $t: t^{*}=1$ to $t^{*}=50(\mathrm{~b})$ magnetic field distribution for various values of time $t$ : $t^{*}=1$ to $t^{*}=50$

The inverse Laplace transform of equations (25)give,

$$
\begin{gathered}
u^{*}\left(y^{*}, t^{*}\right)=\tau_{0}\left(e^{-y^{*}}+1\right) H\left(t^{*}-y^{*}\right) . \\
h^{*}\left(y^{*}, t^{*}\right)=\tau_{0}\left(-1+e^{-y^{*}}\right) H\left(t^{*}-y^{*}\right) . \\
j^{*}\left(y^{*}, t^{*}\right)=\tau_{0}\left[-e^{-y^{*}} H\left(t^{*}-y^{*}\right)+\delta\left(y^{*}-t^{*}\right)\right] .
\end{gathered}
$$

where $H\left(t^{*}-y^{*}\right)$ and $\delta\left(y^{*}-t^{*}\right)$ are, respectively, Heaviside step function and Dirac-delta function. From (26)-(28), the physical significance of the two layers, namely the $k$ layer (Hartmann layer) and $m$ layer (magnetic diffusion layer) can be understood. It is seen that the k layer grows and eventually becomes steady, whereas the $m$ layer diffuses at Alfven speed to infinity. The electric currents generated in the $\mathrm{k}$ layer are returned through $\mathrm{m}$ layer. The figure (5) clearly represent the velocity field, induced magnetic field and the current density in this approximation. The electric currents in the Hartmann layer flow in the $-z$ direction. These currents are returned in a current sheet which propagates in the $y^{*}$ direction at the Alfven speed in our approximation. However the electric currents returned in a small thickness known as Alfven region.

\subsection{Inviscid, Super Conducting Liquid}

In this case, $\nu$ tends to zero and $\sigma$ tends to infinity such that the Prandtl number remains unity. Now we change (13) and (14) to dimensional form and 

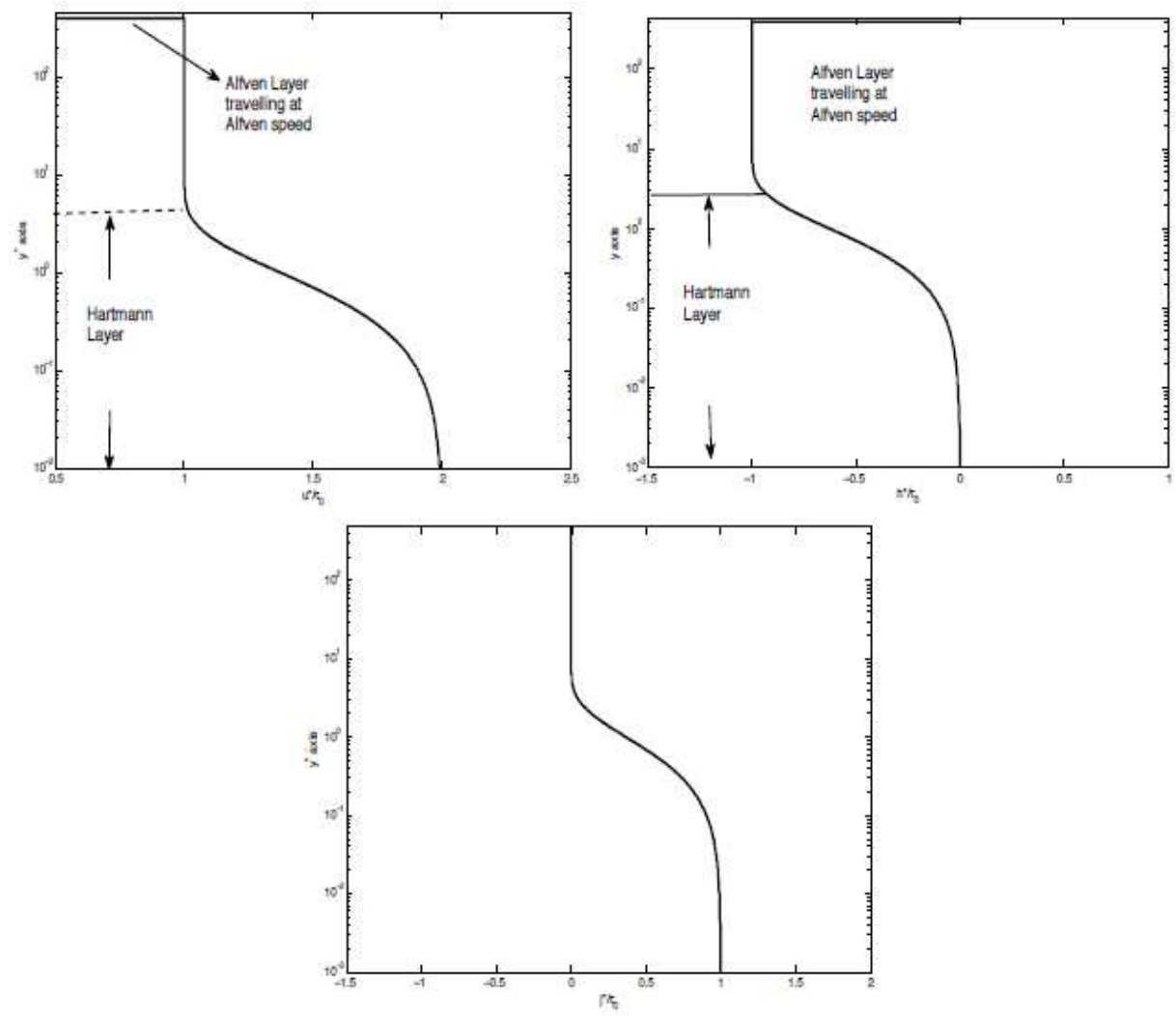

Figure 5: (a) velocity field when $P_{m}$ is unity (for large time), (b) Induced magnetic field when $P_{m}$ is unity (for large time), (c)current density when $P m$ is unity (for large time)

then let $\nu$ tend to zero. We get

$$
\begin{gathered}
\bar{u}=\frac{\tau_{0}}{2 s} e^{-\frac{y}{A} s} \\
\bar{h}=-\frac{\tau_{0}}{2 s} e^{-\frac{y}{A} s}
\end{gathered}
$$

The inverse transform of (29) and (30) are,

$$
\left.\begin{array}{c}
u=\tau_{0} H\left(t-\frac{y}{A}\right) \\
h=-\tau_{0} H\left(t-\frac{y}{A}\right)
\end{array}\right\}
$$

Since the fluid is highly conducting, the magnetic fields are frozen into the fluid. As a result, the electric currents are generated at the surface and at the edge of 
the layer. The layer moves with Alfven speed. The thickness of the Hartmann layer tends to zero. In this limiting case, there is a vortex sheet at $y=0$ and there are two electric current sheets, one at $y=0$, and the other at $y=A t$.

\section{Magnetic Prandtl Number Smaller than Unity $p_{m} \ll 1$}

In this case, we let magnetic Prandtl number much smaller than unity. The values of $\lambda_{1}, \lambda_{2}, D, c_{1}, c_{2}$ are,

$$
\begin{aligned}
& \lambda_{1} \lambda_{2}=s \sqrt{p_{m}} ; \lambda_{1}^{2}+\lambda_{2}^{2}=s\left(1+p_{m}\right)+p_{m} \\
& \lambda_{1}^{2}-\lambda_{2}^{2}=\sqrt{s^{2}\left(1-p_{m}\right)+2 s p_{m}\left(1+p_{m}\right)+p_{m}^{2}} \\
& \lambda_{1}=\sqrt{s+p_{m}} ; D=-s^{2}\left(s+p_{m}\right) ; c_{1}=\frac{\tau_{0}}{s \sqrt{\left(s+p_{m}\right)}} ; \\
& c_{2}=-\frac{\tau_{0} p_{m}^{3 / 2}}{s\left(s+p_{m}\right)^{3 / 2}} ; \lambda_{2}=\frac{s \sqrt{p_{m}}}{\sqrt{s+p_{m}}}
\end{aligned}
$$

The value for $\lambda_{2}$ can also be obtained directly by neglecting viscous term in the equation (9). The resulting equations, which are valid in $m$ layer may be solved to get $\lambda_{2}$. The solutions of $\bar{u}$ and $\bar{h}$ are

$$
\left.\begin{array}{l}
\bar{u}^{*}=\frac{\tau_{0}}{s \sqrt{\left(s+p_{m}\right)}}\left[e^{-\sqrt{s+p_{m}} y^{*}}+\frac{p_{m}^{3 / 2}}{\left(s+p_{m}\right)} e^{-\frac{s \sqrt{p_{m}}}{\sqrt{\left(s+p_{m}\right)}} y^{*}}\right] \\
\bar{h}^{*}=-\frac{\tau_{0} p_{m}}{s\left(s+p_{m}\right)}\left[e^{-\sqrt{s+p_{m}} y^{*}}-e^{-\frac{s \sqrt{p_{m}}}{\sqrt{\left(s+p_{m}\right)}} y^{*}}\right]
\end{array}\right\}
$$

In order to invert equations (32), it is convenient to express (32) in a form
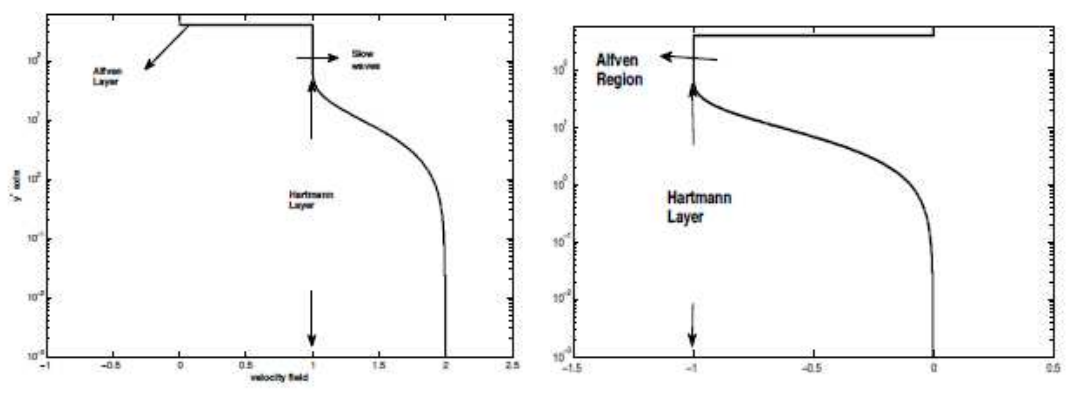

Figure 6: (a)velocity field,(b)Induced magnetic field when $P_{m}$ is $\operatorname{small}\left(P_{m}=0.01\right)$ 
valid for $s \ll p_{m}$. For $s \ll p_{m}$ the velocity and induced magnetic field become, $\bar{u}^{*}=\frac{\tau_{0}}{s}\left[\frac{e^{-\sqrt{p_{m}}\left(1+\frac{s}{2 p_{m}}\right) y^{*}}}{\sqrt{p_{m}}}-\left.e^{-s y^{*}}\right|^{\text {and } \bar{h}^{*}}=\frac{\tau_{0}}{s}\left[e^{-\sqrt{p_{m}} y^{*}} e^{-\frac{s}{2 \sqrt{p_{m}}} y^{*}}-e^{-s y^{*}}\right]\right.$

The inverse transforms of (33) are,

$$
\left.\begin{array}{r}
u^{*}\left(y^{*}, t^{*}\right)=\frac{\tau_{0}}{\sqrt{p_{m}}}\left[e^{-\sqrt{p_{m}} y^{*}} H\left(t^{*}-\frac{y^{*}}{2 \sqrt{p_{m}}}\right)+\sqrt{p_{m}} H\left(t^{*}-y^{*}\right)\right] \\
h^{*}\left(y^{*}, t^{*}\right)=-\tau_{0}\left[-e^{-\sqrt{p_{m}} y^{*}} H\left(t^{*}-\frac{y^{*}}{2 \sqrt{p_{m}}}\right)+H\left(t^{*}-y^{*}\right)\right]
\end{array}\right\}
$$

From (34), it can be seen that, the solutions of $u^{*}$ and $h^{*}$ comprise of slow and fast moving waves. The slow waves are formed in $k$-layer, because, in Hartmann layer, the viscous force is important and it balances the magnetic force. The fast waves are formed in magnetic diffusion layer because it is inviscid to lowest order and the disturbance propagates with Alfven speed. The solution of $u^{*}$ and $h^{*}$ obtained in (34) are plotted in Figure (6) for $P_{m}=0.01$.

\section{Summary and Comments}

The hydrodynamic Rayleigh layer is a diffusing layer since there is no other force to balance viscous diffusion. In the presence of magnetic field, the viscous force can be balanced by magnetic force. Thus, two layers, namely $k$-layer (The Hartmann layer) and $m$-layer (the magnetic diffusion layer) arise. The electric currents generated in $k$-layer are closed through $m$-layer. Thus, while the electric currents in $k$-layer retard the fluid, the returning electric currents in m-layer propel the fluid. It has been shown in Section 3.1.3 that in the limiting case $P_{m}=0$ or $P_{m} \rightarrow 0$ there is no Hartmann layer, we have only the hydrodynamic Rayleigh problem solution for the free surface. The same is the case with Hydromagnetic Rayleigh problem at a rigid surface, where the solution reduces to hydrodynamic Rayleigh problem solution. But, in case rotational effects are included (see: Benton and Loper [2]) the solution corresponding to $P_{m}=0$ does give a transient solution for an Ekman-Hartmann layer.

\section{References}

[1] G.K. Batchelor, An Introduction to Fluid Dynamics, Cambridge University Press, Cambridge, 1993. 
[2] E.R. Benton, D.E. Loper, On the spin-up of an electrically conducting fluid Part 1. The unsteady hydromagnetic Ekman-Hartmann boundary layer problem, J. Fluid Mech., 39 (1969), 561-586.

[3] G.A. Campbell, R.M. Foster, Fourier Integrals for Practical Applications, D. Van Nostrand Company, Inc, New York (1948).

[4] C.C. Chang, J.T. Yen, Rayleigh's problem in magnetohydrodynamics, Phys. Fluids, 2 (1959), 393-403.

[5] D.M. Dix, The magnetohydrodynamic flow past a non-conducting flat plate in the presence of a transverse magnetic field, J. Fluid Mech., 15 (1963), 449-476.

[6] W.F. Hughes, F.J. Young, The Electromagnetodynamics of Fluids, John Wiley \& Sons, New York, 1966.

[7] J. Pedlosky, Geophysical Fluid Dynamics, Springer-Verlag, 1972.

[8] V.J. Rossow, On Rayleigh's problem in magnetohydrodynamics, Phys. Fluids , 3 (1960), 395.

[9] S. Vempaty, R. Balasubramanian, Ekman-Hartmann layer on a free surface, Indian J. Pure appl. Math., 18, No. 5 (1987), 442-450. 
